\title{
Effect of Jerusalem artichoke (Helianthus tuberosus) powder on quality of glass noodles
}

\author{
*Singthong, J. and Thongkaew, C. \\ Department of Agro-Industry, Faculty of Agriculture, Ubon Ratchathani University, Warinchamrap, Ubon \\ Ratchathani, 34190 Thailand
}

\begin{abstract}
Article history:
Received: 21 May 2020

Received in revised form: 3

August 2020

Available Online: 6

December 2020

Keywords:

Jerusalem artichoke,

Glass noodles,

Alginate,

Optimum condition
\end{abstract}

Accepted: 6 November 2020

DOI:

https://doi.org/10.26656/fr.2017.4(S4).004

\begin{abstract}
The Jerusalem artichoke (Helianthus tuberosus) tuber is a vegetable with a low caloric value and a high content of inulin, vitamins and minerals. The purpose of this study was to determine the physicochemical properties of Jerusalem artichoke powder (JA powder). The results indicated that moisture, ash, crude fiber, protein, lipid and carbohydrate contents were $6.71,3.54,2.83,7.58,0.28$ and $79.04 \%$, respectively. The Jerusalem artichoke stored carbohydrates in the form of inulin instead of starch. Total dietary fiber (TDF), soluble dietary fiber (SDF) and insoluble dietary fiber (IDF) of JA powder were 53.11, 40.07 and 13.04\%, respectively. Total sugar, total phenolic compound (TPC) and antioxidant activity by DPPH of JA powder were $0.92 \mathrm{mg}$ fructose/g sample, $6.58 \mathrm{mg}$ GAE/g sample and $93.74 \mathrm{mg}$ TEAC/g sample, respectively. Furthermore, this research reported the design of experimental for optimizing JA powder on the quality of glass noodles using three independent variables: JA powder concentration $(0-3 \%)$, alginate concentration (1.5-2.5\%) and calcium concentration (1-3\%). The optimum condition for the glass noodles was 3\% JA powder, $2 \%$ alginate and 3\% calcium. This optimization revealed that glass noodles had more fiber and sugar, as well as good hardness, cohesiveness, springiness and gumminess. The microstructure of glass noodles addition with JA powder was smaller porosity than glass noodles without JA powder (commercially glass noodle).
\end{abstract}

\section{Introduction}

The Jerusalem artichoke (Helianthus tuberosus) is a plant of the Asteraceae family, which is suitable for cultivation in temperate zones and produces a high yield of edible tubers. The tuber is a high content of inulin, vitamins and minerals, as well as low calories (Saengthongpinit and Sajjaanantakul, 2005; Bach et al., 2012). Jerusalem artichoke stored carbohydrates in the form of inulin instead of starch. Inulin, a non-digestible oligosaccharide, can preferentially stimulate the growth and activity of one or a limited number of desired bacteria in the colon. Inulin in Jerusalem artichoke has been shown to have a prebiotic effect in humans. In addition, inulin also affects blood glucose attenuation, lipid homeostasis, mineral bioavailability and immunomodulatory effects. Including inulin also helps to improve rheological characteristics and nutritional properties of food in term of functional food (Lingyun et al., 2007; Bach et al., 2012). Inulin has been increasingly used in various foods due to its beneficial nutritional attributes. Inulin is a linear polymer of D-fructose joined by $b(2 \rightarrow 1)$ linkages and terminated with a D-glucose molecule linked to fructose by an $\mathrm{a}(1 \rightarrow 2)$ bond, as in sucrose (Panchev et al., 2011; Abou-Arab et al., 2011). The inulin and fructooligosaccharides (FOS) are known to provided health benefits like the stimulation of beneficial colon bacteria (prebiotic), reduction in bowel transit time, increasing in mineral absorption, improvement of immune response and prevention of diseases like intestinal infections, colorectal cancers, obesity, cardiovascular diseases and type II diabetes (Rubel et al., 2015). Commercial inulin preparation is mainly made from chicory (Cichorium intybus L.). However, products of other fructans-containing plants such as Jerusalem artichoke become increasingly interesting for application in food as they do not contain bitter taste compounds and therefore constitute a palatable functional ingredient (Rubel, et al., 2015; Judprasong et al., 2018).

Glass noodle is a product from alginate. Alginate is a 
group of naturally occurring polysaccharides, extracted from brown seaweed. Commercial applications for alginate in food are based on the interaction between alginate and cation to generate or modify food rheology, usually by the formation of a gel network in the presence of calcium ions (Imerson, 1997). The formation of glass noodle product often involves the addition of alginate, which could be used to improve stability especially in avoiding syneresis and to obtain specific textural characteristics (Li et al., 2017). As consumers' demand for variety and healthier food choices are increasing rapidly, food industry and researchers have to continuously improve the variety, quality and availability of food products (Polaki et al., 2010). The objective of this study was to determine the physicochemical properties of Jerusalem artichoke and was to optimize the Jerusalem artichoke on quality of glass noodles.

\section{Material and methods}

\subsection{Raw materials}

The Jerusalem artichoke (Helianthus tuberosus) tubers were obtained from a local farmer at Ubon Ratchathani province, Thailand. The average temperature for the growing season was $28^{\circ} \mathrm{C}$. The Jerusalem artichoke tubers were harvested at maturity (20 weeks). All tubers were washed, peeled, sliced (2 $\mathrm{mm}$ thickness) and soaked in $0.1 \%(\mathrm{w} / \mathrm{v})$ ascorbic acid for 2-3 mins to inhibit the browning reaction. Then dried at $60^{\circ} \mathrm{C}$ until for $3 \mathrm{hrs}$. The dried tubers were milled and ground through 80 mesh to produce JA powder. The JA powder was packed in polyethylene bags and stored in desiccator until requires for further analysis.

\subsection{Physicochemical composition}

Moisture, ash, protein, lipid, crude fiber and carbohydrate of JA powder were determined according to the method of AOAC (2000). Total sugar was measured using the phenol-sulfuric acid assay (Dubois et al., 1956). Total dietary fiber (TDF), soluble dietary fiber (SDF) and insoluble dietary fiber (IDF) were prepared according to the method of AOAC enzymaticgravimetric with slightly modification (AOAC, 1995).

Color changes in the sample were determined by Hunter Lab (Color Flex Model 45/0, USA) in L*, $a^{*}, b^{*}$ color parameters.

Total soluble phenolic constituents of JA powder were determined using the Folin-Ciocalteu reagent with gallic acid as standard. Sample of $20 \mu \mathrm{L}$ was added into a $1.5 \mathrm{~mL}$ cuvette, to which $1.58 \mathrm{~mL}$ of water and $100 \mu \mathrm{L}$ Folin-Ciocalteu reagent was added. The sample was thoroughly mixed and incubated for $5 \mathrm{~min}$ at room temperature. Following incubation $300 \mu \mathrm{L}$ of the $\mathrm{Na}_{2} \mathrm{CO}_{3}(2 \% \mathrm{w} / \mathrm{v})$ solution was added and the mixture was allowed to stand at room temperature for $2 \mathrm{hrs}$. Absorbance was measured at $765 \mathrm{~nm}$ (Spectrophotometer, Model Evolution 300PC, Thermo Fisher Scientific, USA). Results were expressed as gallic acid equivalents (GA) (Oonsivilai et al., 2007; Singthong et al., 2014).

Antioxidant activity was evaluated in accordance with the DPPH method. Free radical scavenging activity was determined using the stable 1,1-diphenyl-2picrylhydrazyl (DPPH) radical according to the procedure described by Brand-Williams et al. (1995) and Singthong et al. (2014) with slight modification. Briefly, $50 \mu \mathrm{L}$ for sample solution was added to $950 \mu \mathrm{L}$ DPPH methanolic solution. The reaction mixture was covered and left in the dark at room temperature. After 15 mins, the absorption was measured at $515 \mathrm{~nm}$. Ascorbic acid was used as a standard control.

\subsection{Optimization}

The experimental design of optimizing JA powder on quality of glass noodles was carried out under various conditions, according to a central composite design (CCD). The effect of three independent variables, JA powder concentration (1-3\%), alginate concentration (1.5 $-2.5 \%)$ and calcium concentration (1-3\%) were determined (Table 1). The independent variables were coded to three levels of $-1,0$ and +1 . The optimization

Table 1. Central composite design (CCD) for the optimization of JA powder on quality of glass noodles

\begin{tabular}{ccccccc}
\hline \multirow{2}{*}{ Treatment } & \multicolumn{3}{c}{ Coded variable $^{\mathrm{a}}$} & \multicolumn{3}{c}{ Process variable $^{\mathrm{b}}$} \\
\cline { 2 - 7 } & $\mathrm{X}_{1}$ & $\mathrm{X}_{2}$ & $\mathrm{X}_{3}$ & $\mathrm{X}_{1}$ & $\mathrm{X}_{2}$ & $\mathrm{X}_{3}$ \\
\hline 1 & -1 & -1 & -1 & 1 & 1.5 & 1 \\
2 & -1 & -1 & 1 & 1 & 1.5 & 3 \\
3 & -1 & 1 & -1 & 1 & 2.5 & 1 \\
4 & -1 & 1 & 1 & 1 & 2.5 & 3 \\
5 & 1 & -1 & -1 & 3 & 1.5 & 1 \\
6 & 1 & -1 & 1 & 3 & 1.5 & 3 \\
7 & 1 & 1 & -1 & 3 & 2.5 & 1 \\
8 & 1 & 1 & 1 & 3 & 2.5 & 3 \\
9 & 0 & 0 & 0 & 2 & 2 & 2 \\
10 & 0 & 0 & 0 & 2 & 2 & 2 \\
11 & 0 & 0 & 0 & 2 & 2 & 2 \\
12 & 0 & 0 & 0 & 2 & 2 & 2 \\
13 & -2 & 0 & 0 & 0 & 2 & 2 \\
14 & 2 & 0 & 0 & 4 & 2 & 2 \\
15 & 0 & -2 & 0 & 2 & 1 & 2 \\
16 & 0 & 2 & 0 & 2 & 3 & 2 \\
17 & 0 & 0 & -2 & 2 & 2 & 0 \\
18 & 0 & 0 & 2 & 2 & 2 & 4 \\
\hline
\end{tabular}

${ }^{a}$ Coded level of variables: $+=$ upper level, $-=$ lower level and $0=$ center points

${ }^{b} \mathrm{X}_{1}, \mathrm{X}_{2}$ and $\mathrm{X}_{3}$ were JA powder concentration (\%), alginate concentration (\%) and calcium concentration (\%), respectively. 
condition was determined using response surface methodology. All experimental data were statistically analyzed with Design-Expert ${ }^{\circledR}$ software (Version 6.0.10, Stat-Ease, Inc., Minneapolis, MN).

Total sugar, crude fiber, color and texture were used as criteria for the selection of the optimized conditions. Total sugar was measured using the phenol-sulfuric acid assay (Dubois et al., 1956). Crude fiber was determined according to the method of AOAC (2000). Color changes in the sample were determined by Hunter Lab (Color Flex Model 45/0, USA). The texture of the sample was evaluated in terms of texture profile analysis by a texture analyzer (texture analyzer model LR5K, Lloyd Instruments Ltd, Segensworth, UK) with a cylindrical probe (diameter $1 \mathrm{~cm}$ ) and a test speed of 60 $\mathrm{mm} / \mathrm{min}$.

\subsection{Structural analysis}

For the scanning electron microscopy, samples were coated with gold using a sputter coater, model SPIMODULE $^{\mathrm{TM}}$ Sputter Coat, and examined by a JEOL, JSM-5410LV scanning microscope.

\subsection{Data analysis}

All experiments and analytical measurements were run in triplicate. Means of each parameter were analyzed by analysis of variance (ANOVA). Differences between treatments at the $5 \%(p \leq 0.05)$ level were considered significant.

Data were statistically analyzed using the DesignExpert ${ }^{\circledR}$ software (Version 6.0.10, Stat-Ease, Inc., Minneapolis, MN) to find the suitable condition for the glass noodle with JA powder.

\section{Results and discussion}

\subsection{Physicochemical characteristics of JA powder}

The chemical compositions of JA powder were shown in Table 2. The amounts of moisture, ash, crude fiber, protein, lipid, and carbohydrate contents were 6.71, $3.54,2.83,7.58,0.28$ and $79.04 \%$, respectively. Total dietary fiber (TDF), soluble dietary fiber (SDF) and insoluble dietary fiber (IDF) of JA powder were 53.11, 40.07 and $13.04 \%$, respectively. The carbohydrate was composed mainly of inulin, while the high protein content emphasized the value as a vital source of nutrients (Bach et al., 2012). The quantity of ash assumed importance, as it determined the nutritionally important minerals. It was seen that JA powder contains more soluble dietary fiber than insoluble dietary fiber, which the human body cannot digest in the digestive tract and not providing energy. It was prebiotic properties that were beneficial to the body (Kapusta et al., 2013; Rubel et al., 2015). The results showed that the JA powder was a slightly yellow color. Total sugar, total phenolic compound (TPC) and antioxidant activity by DPPH of JA powder were $0.92 \mathrm{mg}$ fructose $/ \mathrm{g}$ sample, $6.58 \mathrm{mg} \mathrm{GAE} / \mathrm{g}$ sample and $93.74 \mathrm{mg}$ TEAC/g sample, respectively.

Table 2. Physicochemical composition of JA powder

\begin{tabular}{lc}
\hline Chemical composition & \% wet basis \\
\hline Moisture & $6.71 \pm 0.13$ \\
Protein & $7.58 \pm 0.03$ \\
Lipid & $0.28 \pm 0.01$ \\
Ash & $3.54 \pm 0.04$ \\
Crude fiber & $2.83 \pm 0.08$ \\
Carbohydrate & $79.04 \pm 0.15$ \\
Total Dietary Fiber & $53.11 \pm 0.95$ \\
Insoluble Dietary Fiber & $13.04 \pm 0.95$ \\
Soluble Dietary Fiber & $40.07 \pm 0.14$ \\
Total sugar (mg Fructose/g) & $0.92 \pm 0.001$ \\
Total phenolic (mg GAE/g) & $6.58 \pm 0.001$ \\
Antioxidant (mg TEAC/g) & $93.74 \pm 0.93$ \\
Color & \\
L* & $82.39 \pm 0.33$ \\
$\mathrm{a}^{*}$ & $3.43 \pm 0.05$ \\
$\mathrm{~b}^{*}$ & $13.54 \pm 0.08$ \\
\hline
\end{tabular}

Values are given as mean \pm standard deviation from triplicate determination.

Carbohydrate (by different) $=100$ - moisture content-lipidprotein - ash - crude fiber

$\mathrm{mg} \mathrm{GAE} / \mathrm{g}=\mathrm{mg}$ gallic acid equivalent/g sample $\mathrm{mg} \mathrm{TEAC} / \mathrm{g}=\mathrm{mg}$ trolox equivalent antioxidant capacity $/ \mathrm{g}$ sample.

\subsection{Quality of glass noodles}

This research was focused on the optimizing JA powder on the quality of glass noodle. There are three independent variables, 1-3\% concentration of JA powder (X1), the concentration of alginate $1.5-2.5 \%$ (X2) and calcium concentration 1-3\% (X3) are shown in Table 1. The results showed that the appearance of glass noodle with JA powder was similar to the commercial one but with a slightly yellowish tint when the concentration of JA powder increased. The glass noodle with JA powder was evaluated for physicochemical properties (texture, color, crude fiber and total sugar).

\subsubsection{Textural quality}

The textural properties of glass noodle with JA powder were measured in the texture profile analysis (TPA) mode by a texture analyzer. The results found that the hardness of glass noodle with JA powder was 2.19$7.47 \mathrm{~N}$. The hardness was increased when increasing calcium concentration. This result was similar to the research of Lozano-Vazquez, et al. (2015). Increasing calcium concentration resulted in increased hardness due 
to the cross-linking of the alginate-calcium network (Lupo et al., 2015). This result was similar to alginate. The alginate gel formation was emerged from the outer surface of the beads and slowly diffused of calcium ion until forming a three-dimensional structure. Figure 1 shows the effect of concentration of alginate, JA powder and calcium on the hardness of glass noodle. The results showed that increasing alginate and calcium concentration resulted in increased hardness (Figure 1c). When the concentration of JA powder was increased, the hardness of glass noodle increased (Figure 1a and b).
This results revealed that the arrangement was denser due to cross-linking of alginate and calcium in the threedimension network (Chang and Dobashi, 2003).

Cohesiveness is a value that characterizes the cohesion after the sample pressed twice, which indicates the bond strength within the product structure (Bourne, 1982). The cohesiveness of glass noodle with JA powder was about $0.38-0.45$. It was found that the cohesiveness at the same concentration of JA powder and alginate was not significantly different $(p>0.05)$. It was found that it
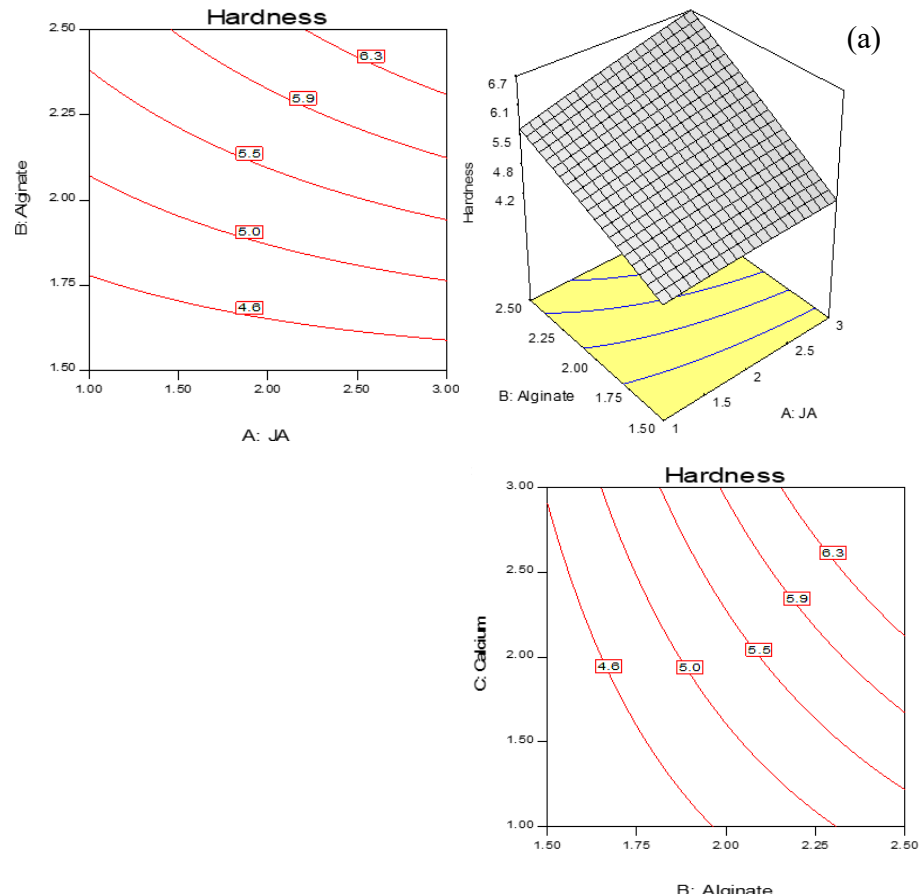

(a)
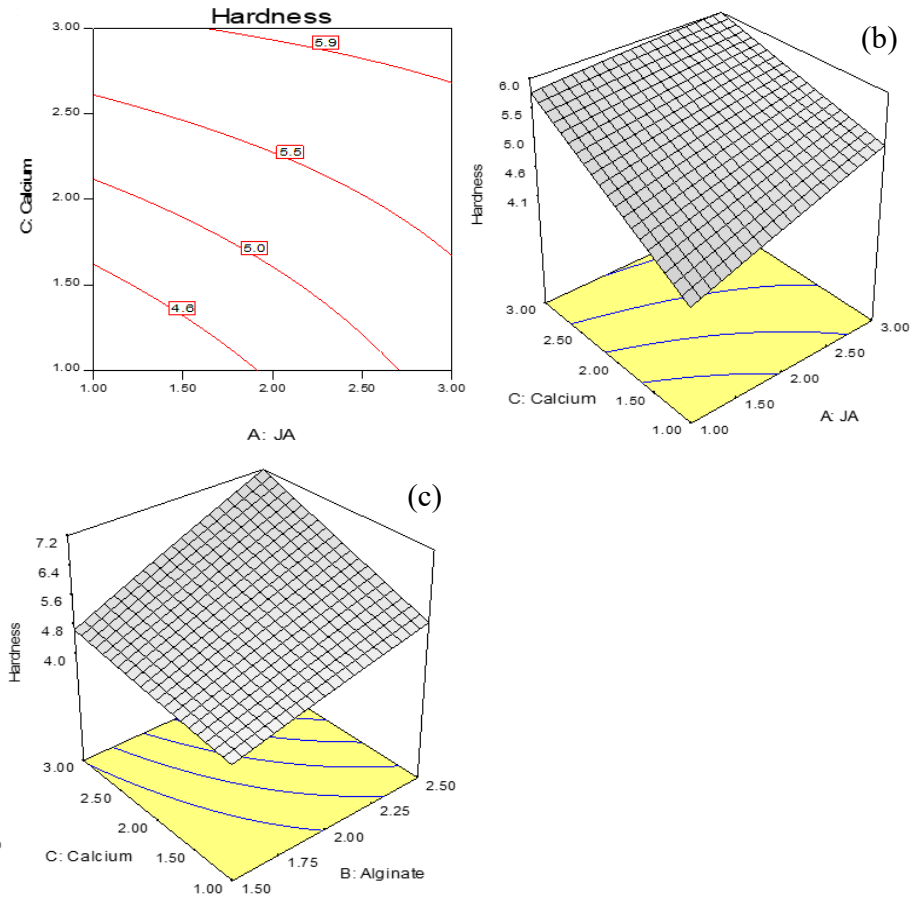

(c)

Figure 1. Response surface plots (2D and 3D) of the effects of the concentration of JA powder, alginate and calcium on hardness. (a) The concentration of JA powder to alginate, (b) The concentration of JA powder to calcium, (c) The concentration of alginate to calcium.
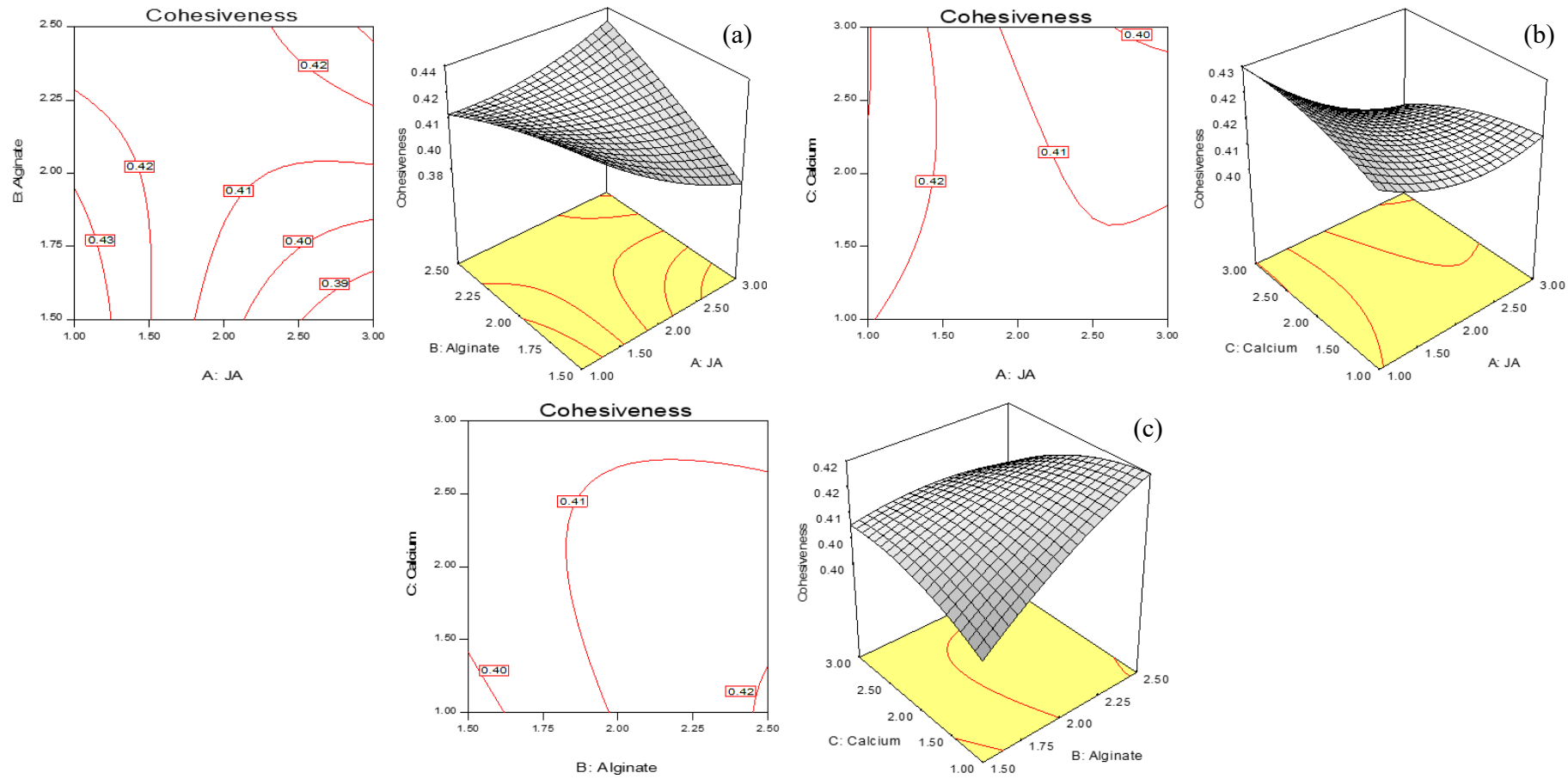

(c)

Figure 2. Response surface plots (2D and $3 \mathrm{D})$ of the effects of the concentration of JA powder, alginate and calcium on cohesiveness. (a) The concentration of JA powder to alginate, (b) The concentration of JA powder to calcium, (c) The concentration of alginate to calcium. 
was statistically significant $(p \leq 0.05)$ due to the JA powder as a core which penetrated the wall of alginate and calcium network structure (Chan et al., 2011). Figure 2 shows the effect of alginate, JA powder and calcium on the cohesiveness of glass noodle. It was found that the concentration of calcium affected the cohesiveness. When the calcium concentration increased, the cohesiveness was also higher. However, when the concentration of JA powder and alginate was reduced, glass noodle was hard and brittle (Figure 2a). Therefore, the cohesiveness values were higher (Walter, 1998). Figure $2 \mathrm{~b}$ showed the concentration ratio of JA powder to calcium. It was found that JA powder and calcium did not affect the cohesiveness because increasing the concentration of the JA powder and calcium did not cause the cohesiveness to increase. When the calcium concentration and the alginate concentration increased, the cohesiveness increased (Figure 2c) because it is related to the internal strength of the bond if the more cross-linking network will increase the bond between alginate and calcium.

Figure 3 shows that when the concentration of JA powder, alginate and calcium increased, the gumminess is also increased. So it required more force to make the sample suitable for swallowing. The gumminess is the denseness that persists throughout mastication or the energy requires to disintegrate a semisolid food to a state ready for swallowing (Bourne, 1982).

The springiness of glass noodle with JA powder was no statistically significant difference $(p>0.05)$. The concentration of JA powder and alginate increased, the springiness of glass noodle increased (Figure 4a). The research has confirmed that JA powder as a core which penetrated the pores of alginate and calcium network structure resulting in greater springiness (Funami et al., 2009; Chan et al., 2011). The concentration of JA powder and calcium did not affect springiness (Figure 4b). Increased alginate and calcium concentrations resulted in increased springiness (Figure 4c) due to cross -linking as a dense structure.

\subsubsection{Color quality}

For the color value, it was found that the lightness color (L*) of glass noodle decreased when the concentration of JA powder increased. The yellow value $\left(b^{*}\right)$ increased when the concentration of JA powder increased. The concentration of alginate and calcium did not affect the lightness but has an effect on yellowness because it has the effect to maintain JA powder as a core material. The effect of the concentration of JA powder, alginate and calcium on the lightness $\left(\mathrm{L}^{*}\right)$ were shown in Figure 5. It was found that decreasing JA powder, alginate and calcium concentrations (Figure 5a and $\mathrm{b}$ ) resulted in increased lightness. Figure $5 \mathrm{c}$ found that increasing calcium concentration resulted in increased lightness but increasing alginate concentration, it did not affect the lightness of glass noodle. On the other hand, when the concentration of JA powder, alginate and calcium increased, it resulted in increased the yellow values of the glass noodle (Figure 6). Because alginate can produce good gel and create a more reticular structure with calcium, resulting in the retention of active compound (JA powder) as a core material (Packer et al.,
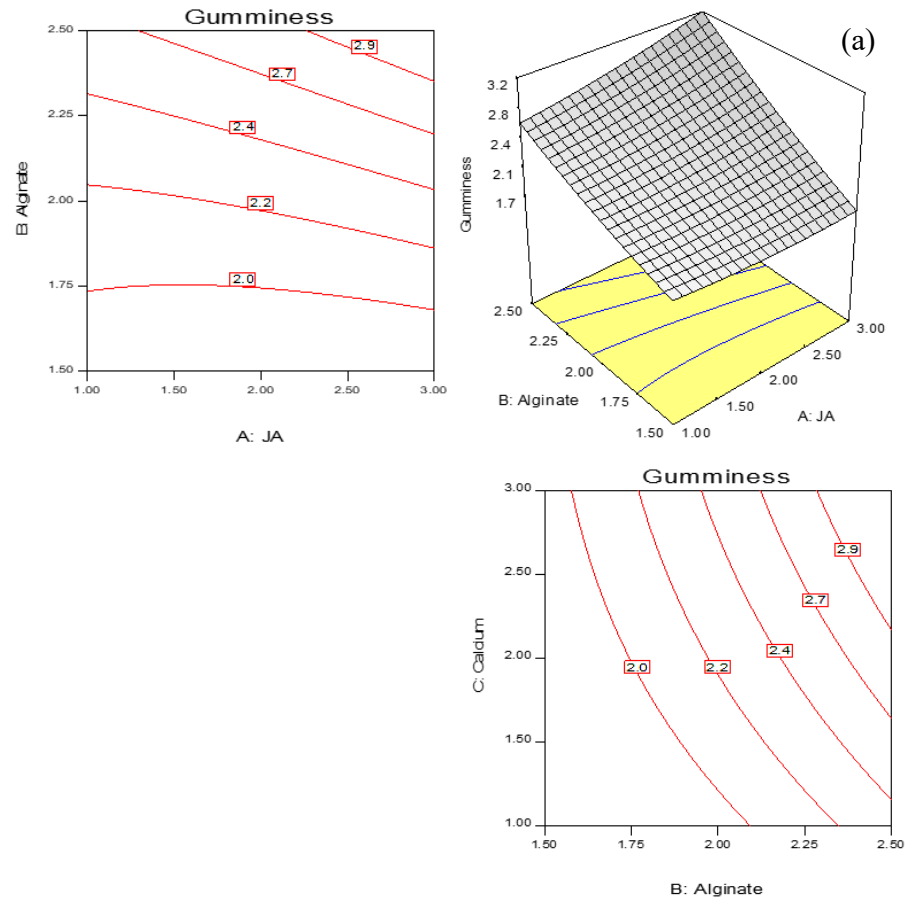
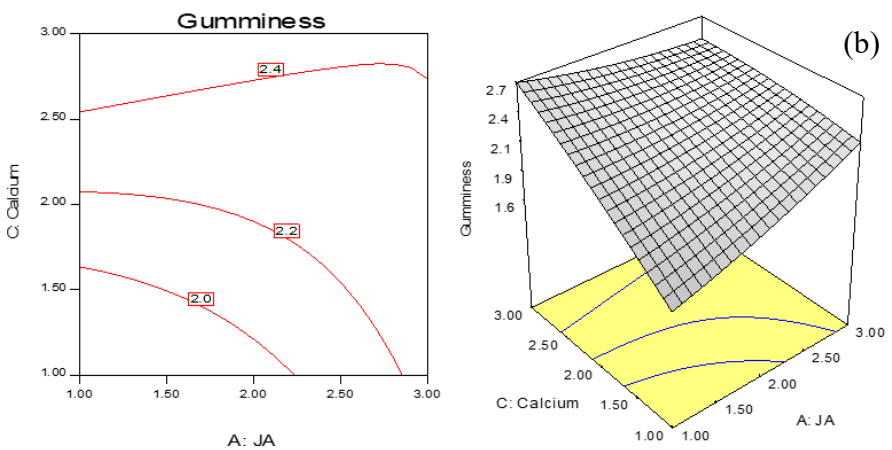

(c)

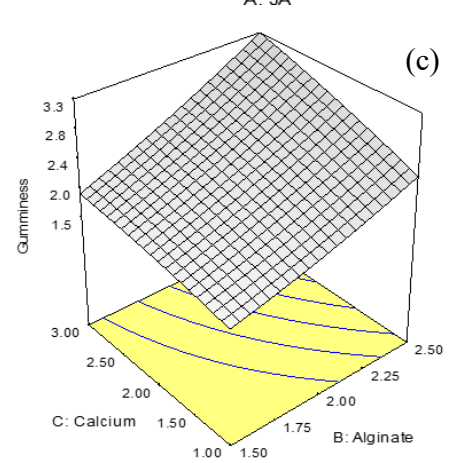

Figure 3. Response surface plots (2D and 3D) of the effects of the concentration of JA powder, alginate and calcium on gumminess. (a) The concentration of JA powder to alginate, (b) The concentration of JA powder to calcium, (c) The concentration of alginate to calcium. 

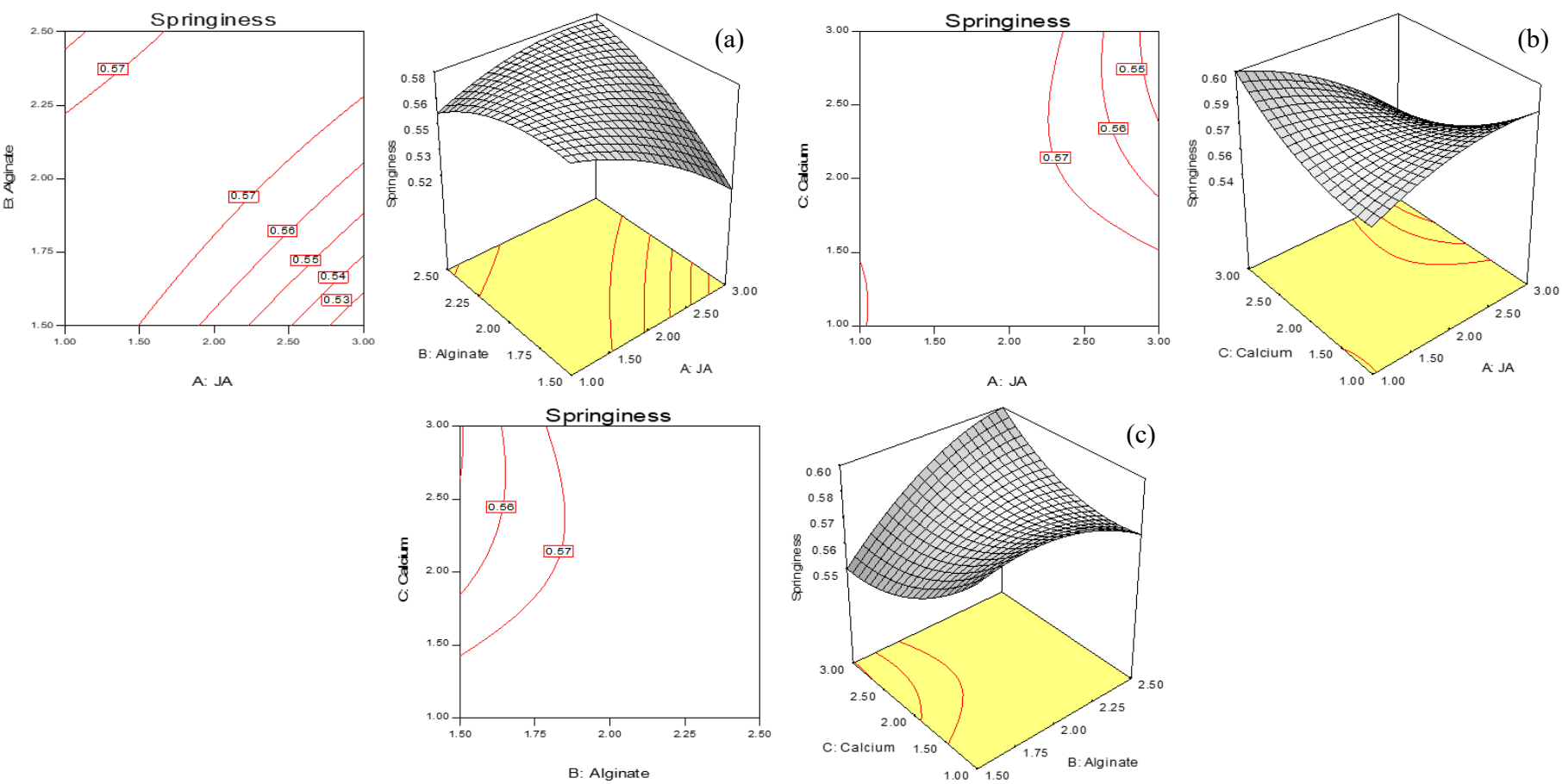

(c)

Figure 4. Response surface plots $(2 \mathrm{D}$ and $3 \mathrm{D})$ of the effects of the concentration of JA powder, alginate and calcium on springiness. (a) The concentration of JA powder to alginate, (b) The concentration of JA powder to calcium, (c) The concentration of alginate to calcium.
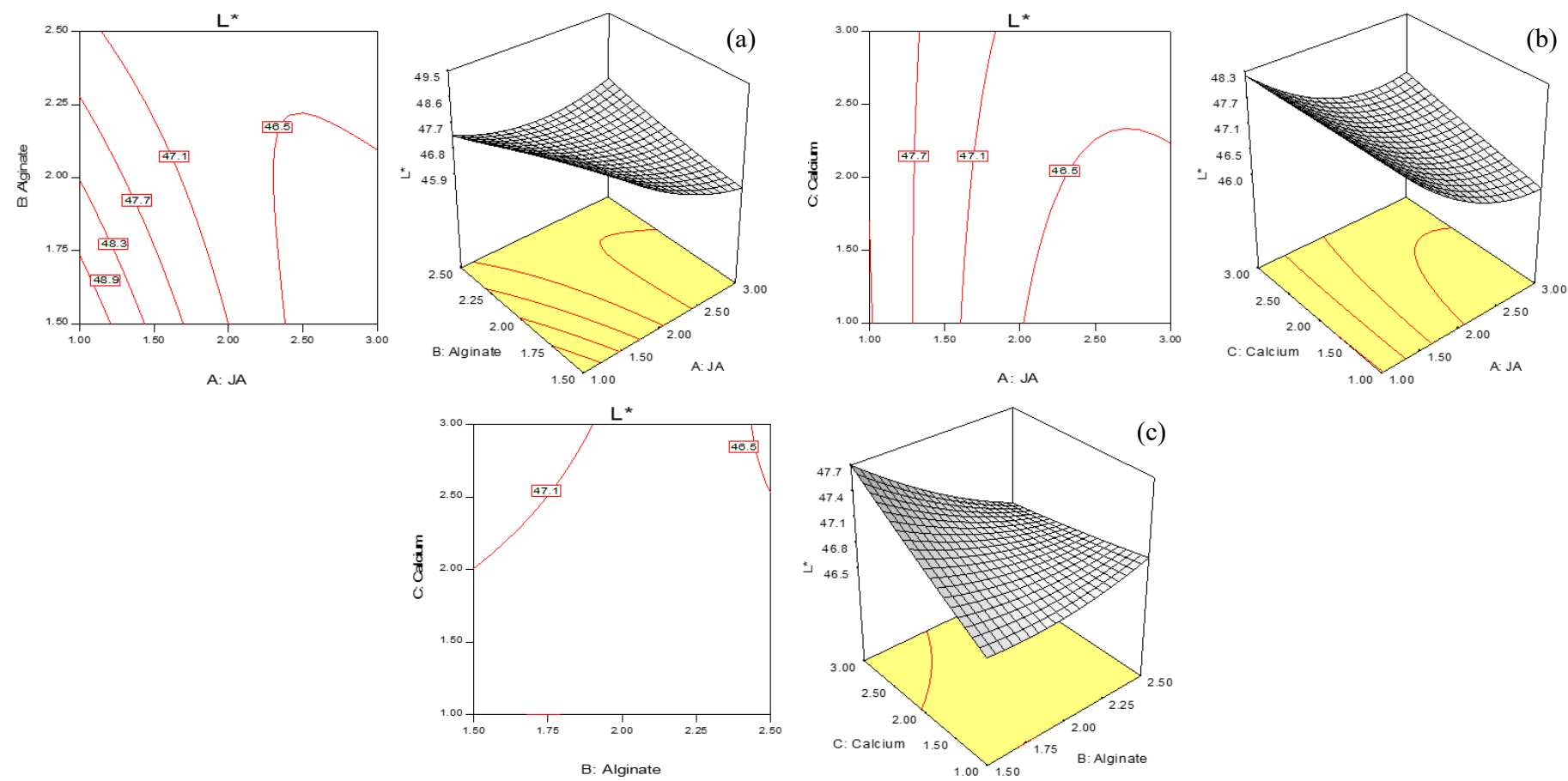

(c)

Figure 5. Response surface plots (2D and 3D) of the effects of the concentration of JA powder, alginate and calcium on lightness (L*). (a) The concentration of JA powder to alginate, (b) The concentration of JA powder to calcium, (c) The concentration of alginate to calcium.

1999).

\subsubsection{Crude fiber and total sugar}

The effect of various factors is the concentration of JA powder, alginate and calcium on the fiber and sugar content were studied as shown in Figure 7 and 8 . Increasing the concentration of JA powder resulted in increased fiber content (Figure 7). Due to the increased in the amount of active compounds needed to be collected the fiber content contained in JA powder as a core material was also increased. In addition, increasing alginate and calcium concentration resulted in increased the structure of the gel and presented the cross-linking of alginate and calcium resulting in a three-dimensional structure, which can store more active compounds (JA powder) (Bartolo et al., 2013; Zeeb et al., 2015).

Figure 8a showed that when the ratio between the JA powder and alginate increased, it resulted in increased total sugar. Alginate acts as a wall material which reduces the loss of active compounds and helps control the release of active compounds (Gharsallaoui et al., 

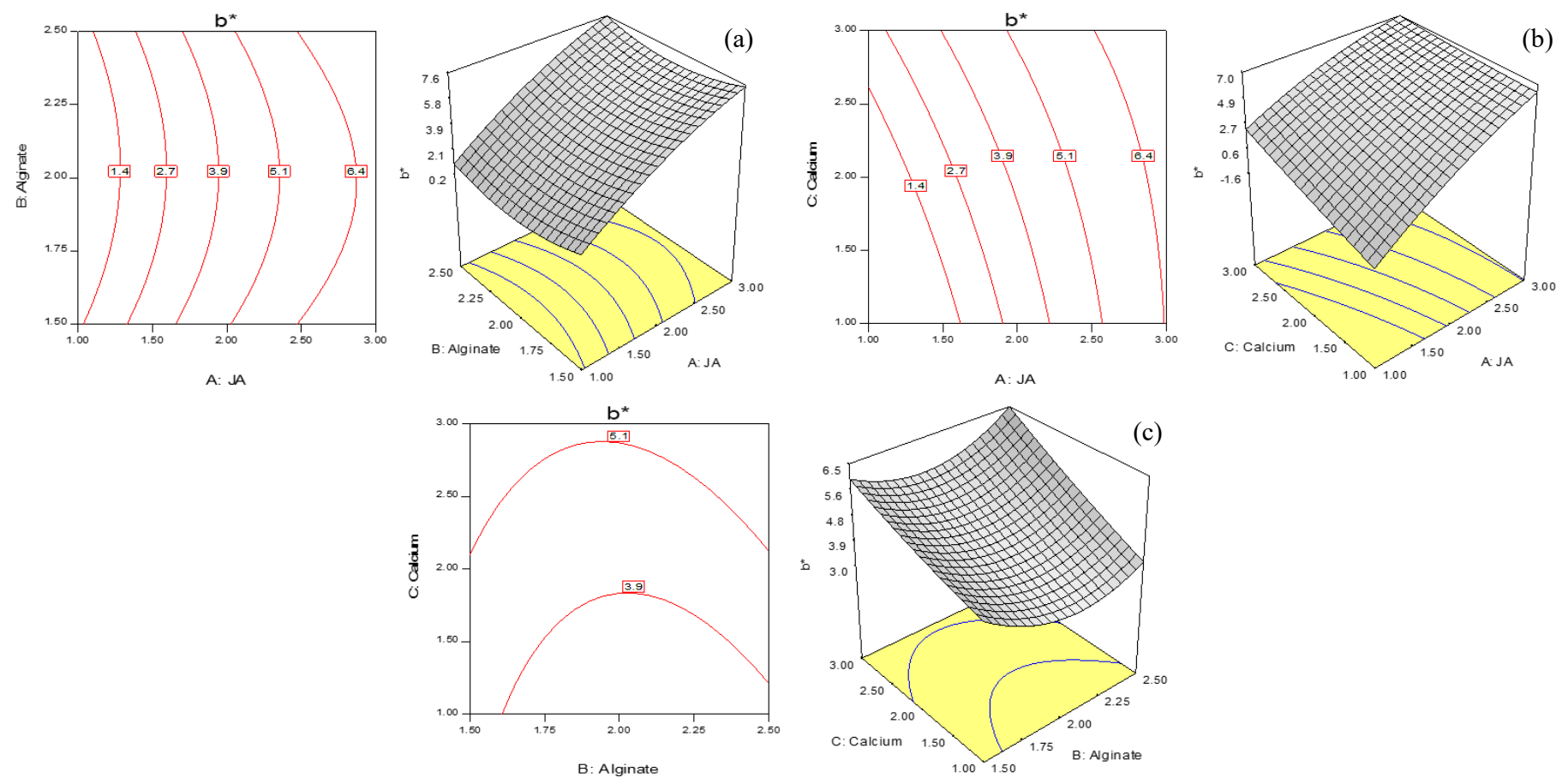

Figure 6. Response surface plots (2D and 3D) of the effects of the concentration of JA powder, alginate and calcium on yellowness ( $b^{*}$ ). (a) The concentration of JA powder to alginate, (b) The concentration of JA powder to calcium, (c) The concentration of alginate to calcium.
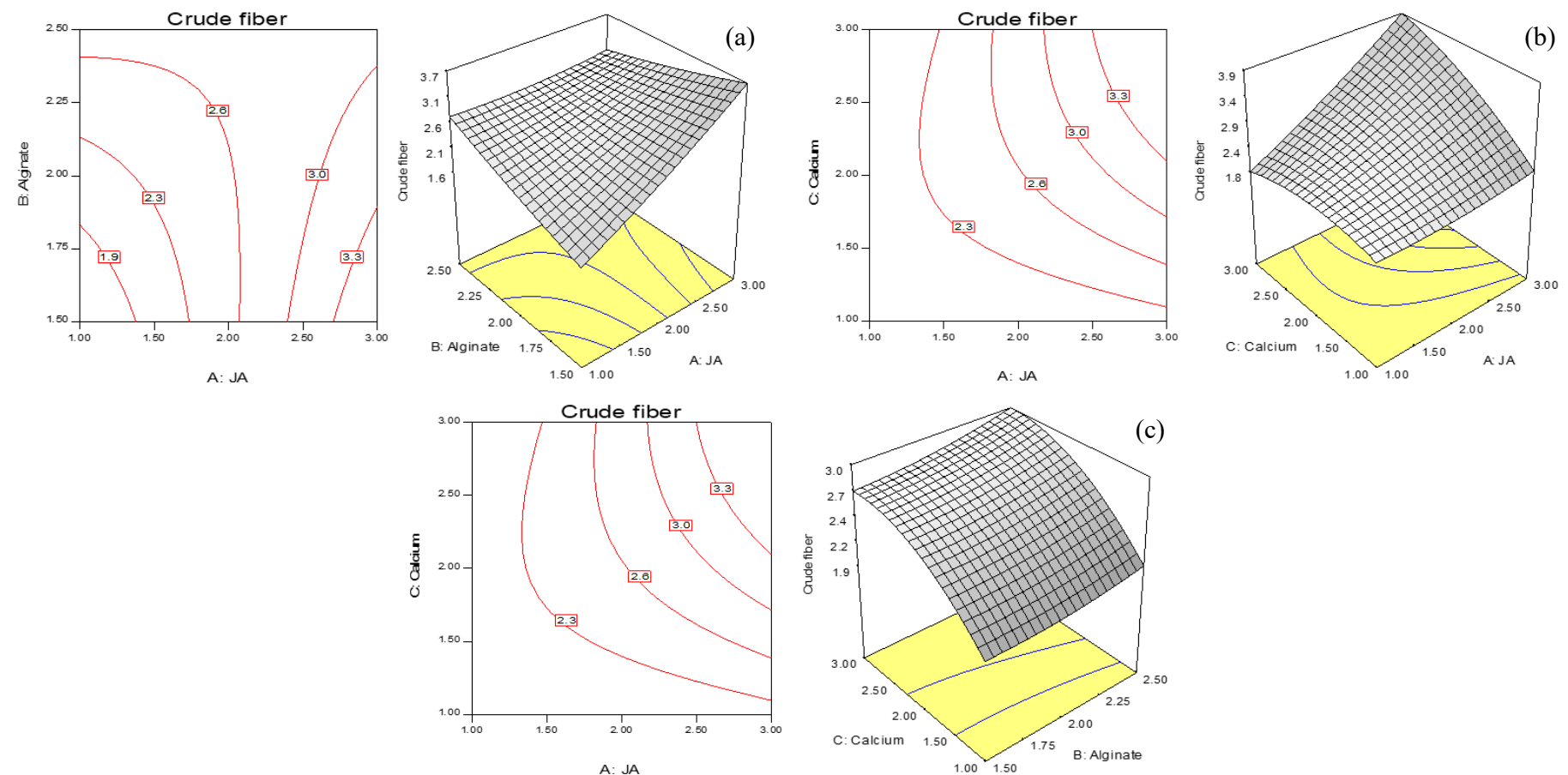

Figure 7. Response surface plots (2D and 3D) of the effects of the concentration of JA powder, alginate and calcium on crude fiber content. (a) The concentration of JA powder to alginate, (b) The concentration of JA powder to calcium, (c) The concentration of alginate to calcium.

2007). When the concentration of JA powder and calcium increased, it resulted in the increased of total sugar (Figure $8 \mathrm{~b}$ ). The concentration of alginate and calcium increased, it resulted in increased in the total sugar (Figure 8c).

\subsubsection{Optimization}

From the statistical analysis with the Design-expert 6.0.10 software to find the optimum condition, it was found that $3 \%$ concentration of JA powder, $2 \%$ alginate concentration and $3 \%$ calcium concentration provided the suitable glass noodle with JA powder. This glass noodle gave the good quality of hardness, cohesiveness, springiness and gumminess including the high amount of crude fiber and total sugar.

\subsection{Microstructure}

The optimum condition using $2 \%$ alginate concentration, $3 \%$ calcium concentration and $3 \%$ of JA powder gave the good quality of glass noodle. After that, the samples were dried using freeze-drying before being studied for structural analysis compared with the normal 

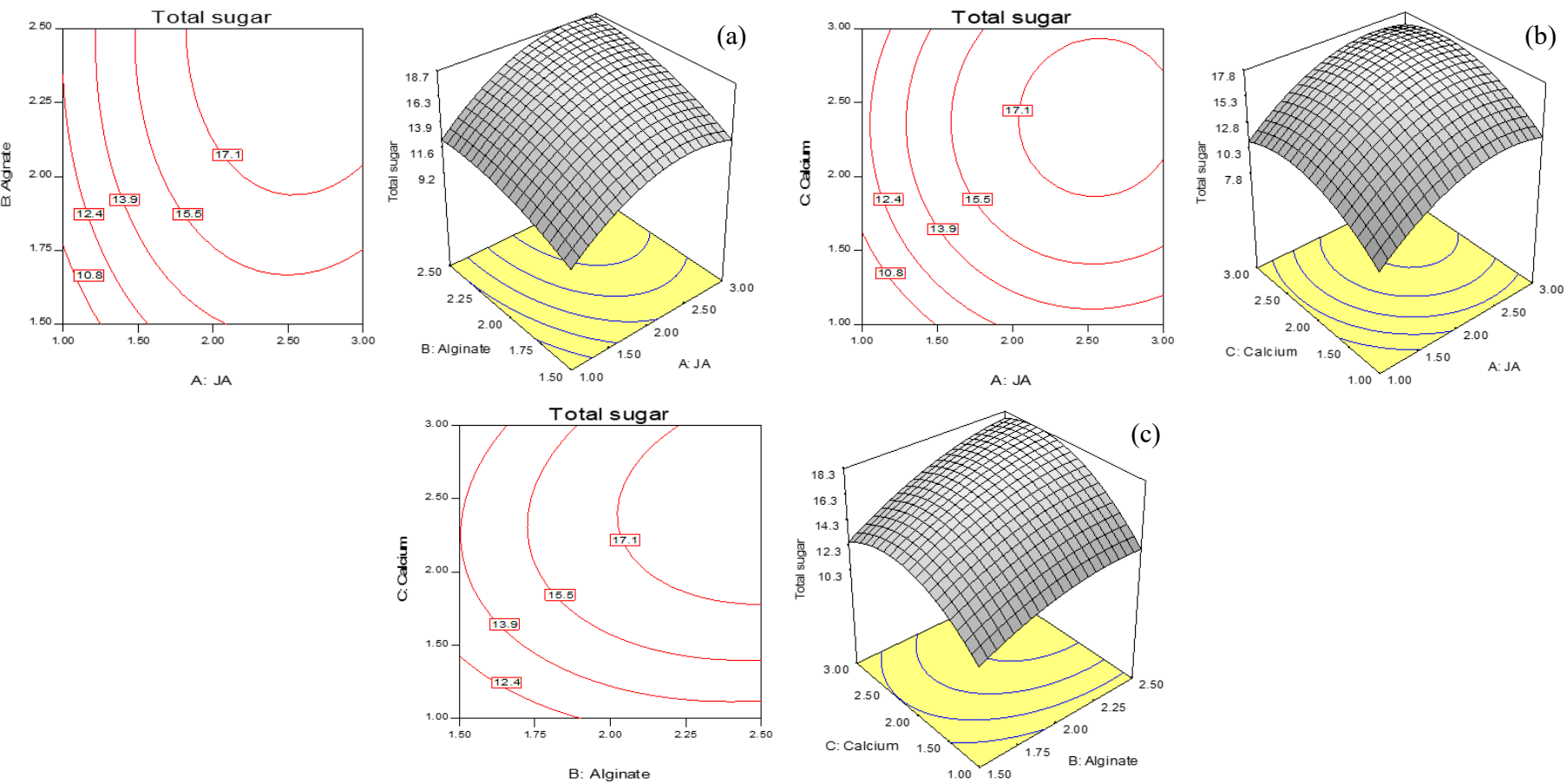

(c)

Figure 8. Response surface plots (2D and 3D) of the effects of the concentration of JA powder, alginate and calcium on total sugar content. (a) The concentration of JA powder to alginate, (b) The concentration of JA powder to calcium, (c) The concentration of alginate to calcium.

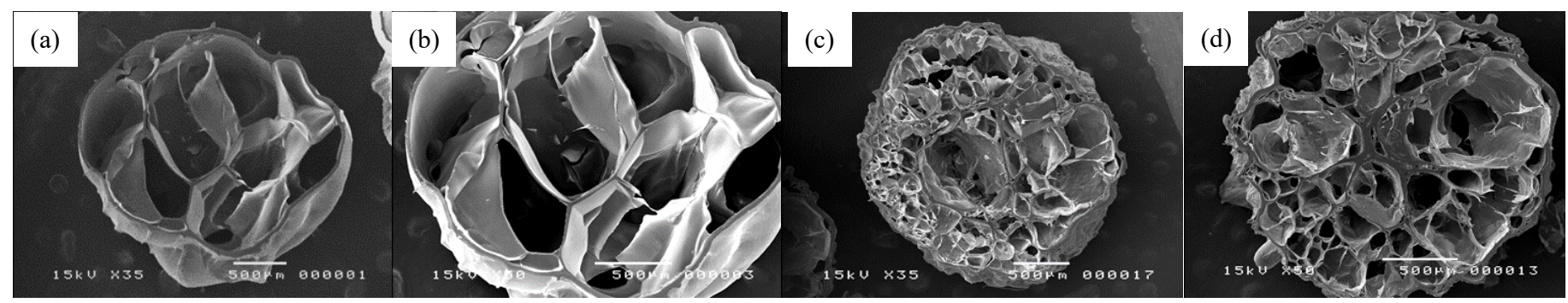

Figure 9. Electron microscope photography of glass noodle. (a) Glass noodle (control) 15KV x35, (b) Glass noodle (control) $15 \mathrm{KV} \times 50$, (c) Glass noodle with JA powder $15 \mathrm{KV}$ x35, (d) Glass noodle with JA powder $15 \mathrm{KV} \times 50$

one without JA powder (control) using scanning electron microscopy (SEM). The results showed that the glass noodle was porous which caused by cross-linking of the alginate gel (Figure 9). When comparing the control and glass noodle with JA powder, it was found that the control displayed with large pores (Figure 9a and b), while the glass noodle with JA powder, the pores that were smaller than the control (Figure 9c and $d$ ). Therefore, adding the JA powder to the glass noodle resulted in decreased a large porosity and more small porosities (Chan et al., 2011).

\section{Conclusion}

JA powder was a high content of dietary fiber and antioxidant. The optimum condition of glass noodle with JA powder was $3 \%$ of JA powder, $2 \%$ alginate and $3 \%$ calcium concentrations. The glass noodle showed the good quality of the hardness, cohesiveness, springiness and gumminess, including high amount of crude fiber. Glass noodle with JA powder was small porous structure. Therefore, improving the glass noodle to have higher nutritional value by using the JA powder makes healthy food and more choices for consumers.

\section{Conflict of interest}

The authors declare no conflict of interest.

\section{Acknowledgments}

The author acknowledges the Financial support by Ubon Ratchathani University for this research.

\section{References}

Abou-Arab, A.A., Talaat, H.A. and Abu-Salem, F.M. (2011). Physico-chemical properties of inulin produced from Jerusalem artichoke tubers on bench and pilot plant scale. Australian Journal of Basic and Applied Science, 5(5), 1297-2011.

AOAC. (1995). Official of Association of Analytical Chemistry $1^{\text {st }}$ ed. Washington D.C., USA: AOAC.

AOAC. (2000). Official of Association of Analytical Chemistry $17^{\text {th }}$ ed. Washington D.C., USA: AOAC.

Bach, V., Kidmose, U., Bjfrn, G.K. and Edelenbos, M. (2012). Effect of harvest time and variety on sensory 
quality and chemical composition of Jerusalem artichoke (Helianthus tuberosus) tubers. Food Chemistry, 133(1), 82-89. https://doi.org/10.1016/ j.foodchem.2011.12.075

Bartolo, P.J., Lagoa, R. and Mendes, A. (2003). Solid Freedom Fabrication Symposium Proceeding. Portugal: School of Technology and Management.

Bourne, M.C. (1982). Food Texture and Viscosity Concept and Measurement. New York: Academic Press. https://doi.org/10.1016/B978-0-12-1190606.50011-3

Brand-Williams, W., Cuvelier, M.E. and Berset, C. (1995). Use of free radical method to evaluate antioxidant activity. LWT - Food Science and Technology, 28(1), 25-30. https://doi.org/10.1016/ S0023-6438(95)80008-5

Chan, E.-S., Wong, S.-L., Lee, P.-P., Lee, J.-S., Ti, T. B., Zhang, Z., Poncelet, D., Ravindra, P., Phan, S-H. and Yim, Z.-H. (2011). Effects of starch filler on the physical properties of lyophilized calcium-alginate beads and the viability of encapsulated cells. Carbohydrate Polymers, 83(1), 225-232. https:// doi.org/10.1016/j.carbpol.2010.07.044

Chang, C.P. and Dobashi, T. (2003). Preparation of alginate complex capsules containing eucalyptus essential oil and its controlled release. Colloids and Surfaces B: Biointerfaces, 32(3), 257-262. https:// doi.org/10.1016/j.colsurfb.2003.07.002

Dubois, M., Gilles, K.A., Hamilton, J.K., Rebers, P.A. and Smith, F. (1956). Colorimetric method for determination of sugar and related substances. Analytical Chemistry, 28(3), 350-356. https:// doi.org/10.1021/ac60111a017

Funami, T., Fang, Y., Noda, S., Ishihara, S., Nakauma, M., Draget, K. I., Nishinari, K. and Phillips, G.O. (2009). Rheological properties of sodium alginate in an aqueous system during gelation in relation to supermolecular structures and $\mathrm{Ca}^{2+}$ binding. Food Hydrocolloids, 23(7), 1746-1755. https://doi.org/10.1016/j.foodhyd.2009.02.014

Gharsallaoui, A., Roudaut, G., Chambin, O., Voilley, A. and Saurel, R. (2007). Applications of spray-drying in microencapsulation of food ingredients: An overview. Food Research International, 40(9), 1107 -1121. https://doi.org/10.1016/j.foodres.2007.07.004

Imerson, A. (1997). Thickening and Gelling Agents for Food. New York: Blackie Academic and Professional. https://doi.org/10.1007/978-1-46152197-6

Judprasong, K., Archeepsudcharit, N., Chantapiriyapoon, K., Tanaviyutpakdee, P. and Temviriyanukul, P. (2018). Nutrients and natural toxic substances in commonly consumed Jerusalem artichoke (Helianthus tuberosus L.) tuber. Food Chemistry, 238, $\quad$ 173-179.

https://doi.org/10.1016/

j.foodchem.2016.09.116

Kapusta, I., Krok, E.S., Jamro, D.B., Cebulak, Y., Kaszuba, J. and Salach, R.T. (2013). Identification and quantification of phenolic compounds from Jerusalem artichoke (Helianthus tuberosus L.) tubers. Journal of Agriculture and Environment, 11 (3), 601-606.

Li, Q-Q., Wang Y-S., Chen H-H., Liu, S. and Li, M. (2017). Retardant effect sodium alginate on the retrogradation properties of normal cornstarch and anti-retrogradation mechanism. Food Hydrocolloids, 69, 1-9. https://doi.org/10.1016/ j.foodhyd.2017.01.016

Lingyun, W., Jianhua, W., Xiaodong, Z., Da, T., Yalin, Y., Chenggang, C., Tianhua, F. and Fan, Z. (2007). Studied on the extracting technical conditions of inulin from Jerusalem artichoke tubers. Journal of Food Engineering, 79(3), 1087-1093. https:// doi.org/10.1016/j.jfoodeng.2006.03.028

Lozano-Vazquez, G., Lobato-Calleros, C., EscalonaBuendia, H., Chavez, G., Alvarez-Ramirez, J., and Vernon-Carter, E.J. (2015). Effect of the weight ratio of alginate-modified tapioca starch on the physicochemical properties and release kinetics of chlorogenic acid containing beads. Food Hydrocolloids, 48, 301-311. https://doi.org/10.1016/ j.foodhyd.2015.02.032

Lupo, B., Maestro, A., Gutiérrez, J.M. and González, C. (2015). Characterization of alginate beads with encapsulated cocoa extract to prepare functional food: Comparison of two gelation mechanisms. Food Hydrocolloids, 49, 25-34. https://doi.org/10.1016/ j.foodhyd.2015.02.023

Oonsivilai, R., Cheng, C., Bomser, J., Ferruzzi, M. and Ningsanond, S. (2007). Phytochemical profiling and phase II enzyme-inducing properties of Thunbergia laurifolia Lindl. (RC) extracts. Journal of Ethnopharmacology, 114(3), 300-306. https:// doi.org/10.1016/j.jep.2007.08.015

Packer, L., Rimbach, G. and Virgili, F. (1999). Antioxidant activity and biologic properties of a procyanidin-rich extract from pine (Pinus maritima) bark, pycnogenol. Free Radical Biology and Medicine, 27(5-6), 704-724. https://doi.org/10.1016/ S0891-5849(99)00090-8

Panchev, I., Delchev, N., Kovacheva, D. and Slavov, A. (2011). Physicochemical characteristics of inulins obtained from Jerusalem artichoke (Helianthus tuberosus L.). European Food Research and Technology, 233, 889. https://doi.org/10.1007/ 
s00217-011-1584-8

Polaki, P., Xasapis, P., Fasseas, C., Yanniotis, S. and Mandala, I. (2010). Fiber and hydrocolloid content affect the microstructural and sensory characteristics of fresh and frozen stored beads. Journal of Food Engineering, 97(1), 1-7. https://doi.org/10.1016/ j.jfoodeng.2009.04.031

Rubel, I.A., Perez, E.E., Manrique, G.D. and Genovese, D.B. (2015). Fibre rnrichment of wheat bread with Jerusalem artichoke inulin: Effect on dough rheology and bread quality. Food Structure, 3, 21-29. https:// doi.org/10.1016/j.foostr.2014.11.001

Saengthongpinit, W. and Sajjaanantakul, T. (2005). Influence of harvesting time and storage temperature on characteristics of inulin from Jerusalem artichoke (Helianthus tuberosus L.) tubers. Postharvest Biological and Technology, 37(1), 93-100. https:// doi.org/10.1016/j.postharvbio.2005.03.004

Singthong, J., Oonsivilai, R., Oonmetta-aree, J. and Ningsanond, S. (2014). Bioactive compounds and encapsulation of Yanang (Tiliacora Triandra) leaves. African Journal of Traditional, Complementary and Alternative Medicines, 11, 76 84. https://doi.org/10.4314/ajtcam.v11i3.11

Walter, H.R. (1998). Polysaccharide Association Structures in Food. New York, USA: Marcel Dekker, Inc. https://doi.org/10.1201/b13242

Zeeb, B., Saberi, A.H., Weiss, J. and McClements, D.J. (2015). Formation and characterization of filled hydrogel beads based on calcium alginate: Factors influencing nanoemulsion retention and release. Food Hydrocolloids, 50, 27-36. https:// doi.org/10.1016/j.foodhyd.2015.02.041 